\title{
Editorial
}

\section{Coal and Biomass Combustion}

\author{
Hanhui Jin $\left(\mathbb{D},{ }^{1}\right.$ Kun Luo, ${ }^{2}$ Oliver Stein, ${ }^{3}$ Hiroaki Watanabe $\mathbb{D D}^{4},{ }^{4}$ and Xiaoke Ku $\left(\mathbb{D}{ }^{1}\right.$ \\ ${ }^{1}$ School of Aeronautics and Astronautics, Zhejiang University, Hangzhou 310027, China \\ ${ }^{2}$ State Key Laboratory of Clean Energy Utilization, Zhejiang University, Hangzhou 310027, China \\ ${ }^{3}$ Institut für Technische Verbrennung, Universität Stuttgart, Herdweg 51, 70174 Stuttgart, Germany \\ ${ }^{4}$ Department of Mechanical Engineering, Kyushu University, Fukuoka, Fukuoka 8190395, Japan
}

Correspondence should be addressed to Hanhui Jin; enejhh@zju.edu.cn

Received 8 October 2018; Accepted 8 October 2018; Published 29 November 2018

Copyright @ 2018 Hanhui Jin et al. This is an open access article distributed under the Creative Commons Attribution License, which permits unrestricted use, distribution, and reproduction in any medium, provided the original work is properly cited.

Coal combustion is the largest source of global energy consumption and electricity generation worldwide now and will remain so in the foreseeable future, although coal is also one of the major sources of air pollution. Increasing the efficiency of coal-fired power plants across the world will greatly reduce air pollution and extend the lifetime of our coal resources. The combustion of solid biomass fuels as a renewable energy source has grown significantly in the last decade, principally because it can be used to replace fossil fuels (coal, oil, and natural gas).

For this special issue of the Journal of Combustion, we have invited researchers to focus on the combustion of solid fuels and their related processes in power generation. The submitted papers cover a diversity of aspects reflecting the latest progress in the field. These include integrating the supercritical $\mathrm{CO}_{2}$ Brayton cycle with the coal-fired circulating fluidized-bed boiler, coal and biomass cofiring systems, combustion kinetics of biomass materials, thermal improvement and combustion kinetics of enriched coal, and Computed Tomography of Chemiluminescence (CTC) for turbulent industrial flame reconstruction.

Biomass appears to be a promising source of power generation and about half of the papers focus on the biomass related fields, including the combustion kinetic characteristics of wood powder and pellets, as well as the combustion process. Although some methods to utilize pure biomass have been developed (e.g., pyrolysis, gasification, and combustion), the coal and biomass cofiring system is still the most important technology for biomass energy conversion. In this special issue, both of the final published papers on biomass utilization pay attention to the coal and biomass cofiring process, implying that coal combustion is still difficult to be entirely replaced in energy generation.

Regarding the methods used in the published research, both experimental and numerical methods show their advantages in different fields. In this special issue, the combustion kinetics of different materials were studied experimentally and numerical research was carried out to study the combustion processes. Computed Tomography of Chemiluminescence (CTC) for turbulent industrial flame reconstruction was also included in the current issue, which may become a useful tool for researchers and scientists for studying flame structure and evolution.

\section{Conflicts of Interest}

The members of the guest editorial team do not have any possible conflicts of interest or private agreements with companies, including the following. (1) Any of us will refuse to handle the manuscript, if he has recently coauthored with the author of the manuscript, which could be perceived to be influenced by the relationship or recently shared an affiliation or employment history with the author. (2) We will identify any undeclared conflicts that an author might have according to our knowledge of the discipline, when making a recommendation on the manuscript. (3) We will consider potential conflicts when assigning the manuscript to reviewers according to our knowledge. We will not select a referee who (i) works or has recently worked at the same institution 
as the author or authors, (ii) has recently coauthored a paper with the author or authors, or (iii) has a recent or current collaboration with the author. (4) If we have concerns about a potential reviewer, we will consider appointing someone else. If we believe a reviewer's recommendation on a manuscript was made to further their own interests, we may tell the authors they do not need to address that point.

Hanhui Jin

Kun Luo

Oliver Stein

Hiroaki Watanabe

Xiaoke Ku 


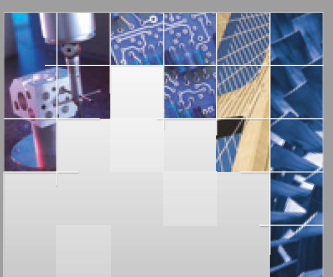

\section{Enfincering}
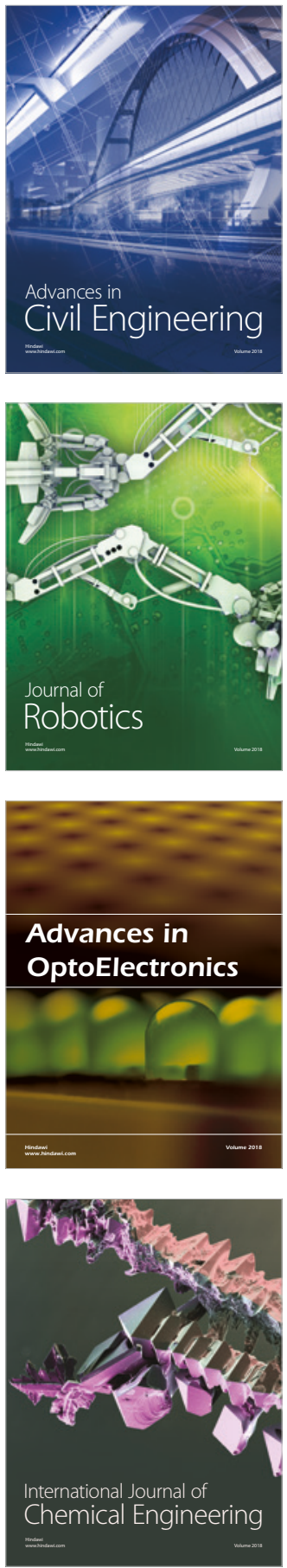

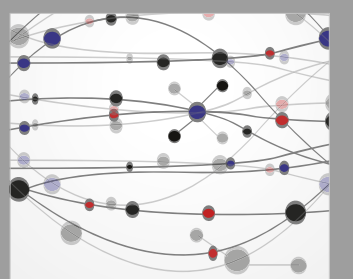

\section{Rotating \\ Machinery}

The Scientific World Journal

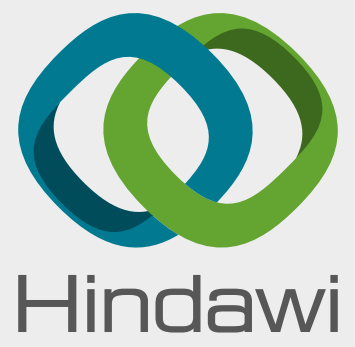

Submit your manuscripts at

www.hindawi.com
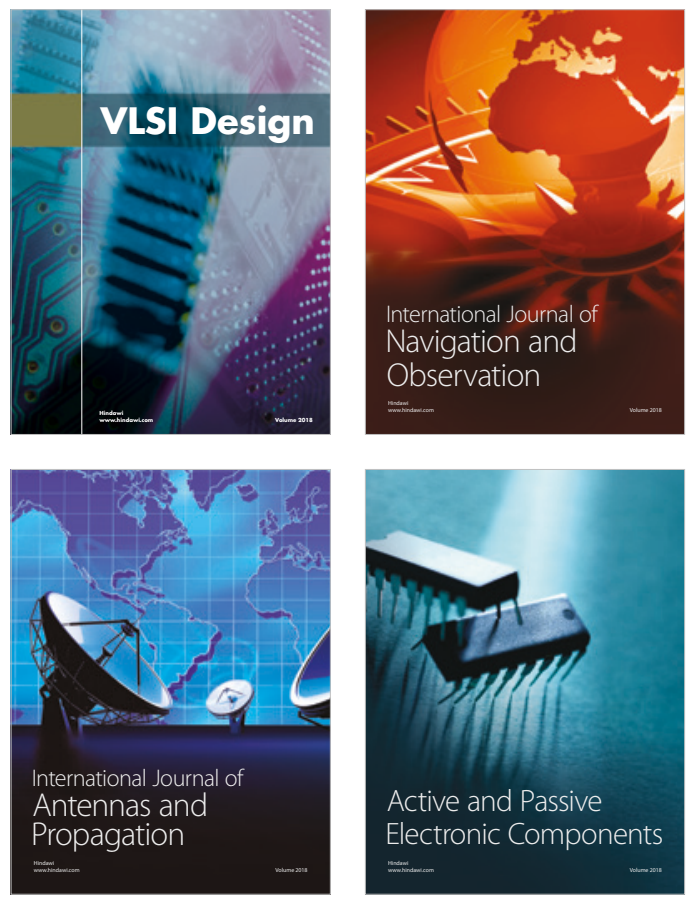
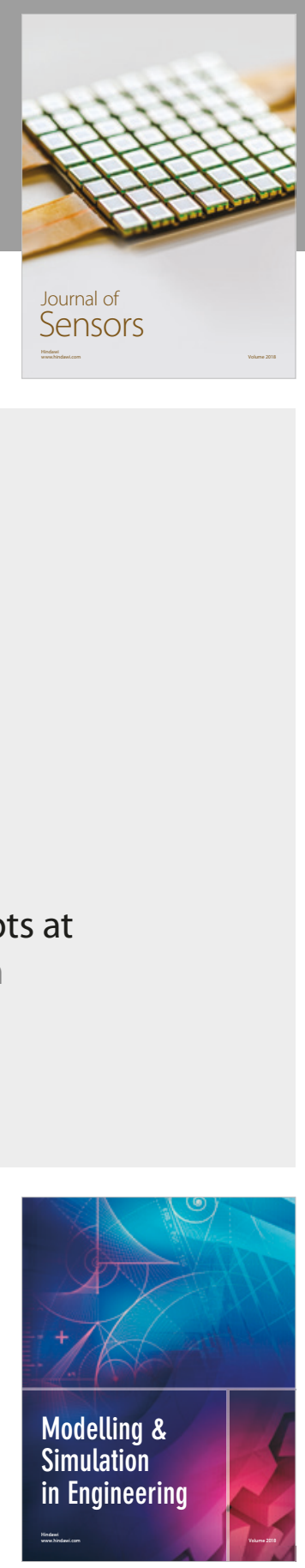

\section{Advances \\ Multimedia}
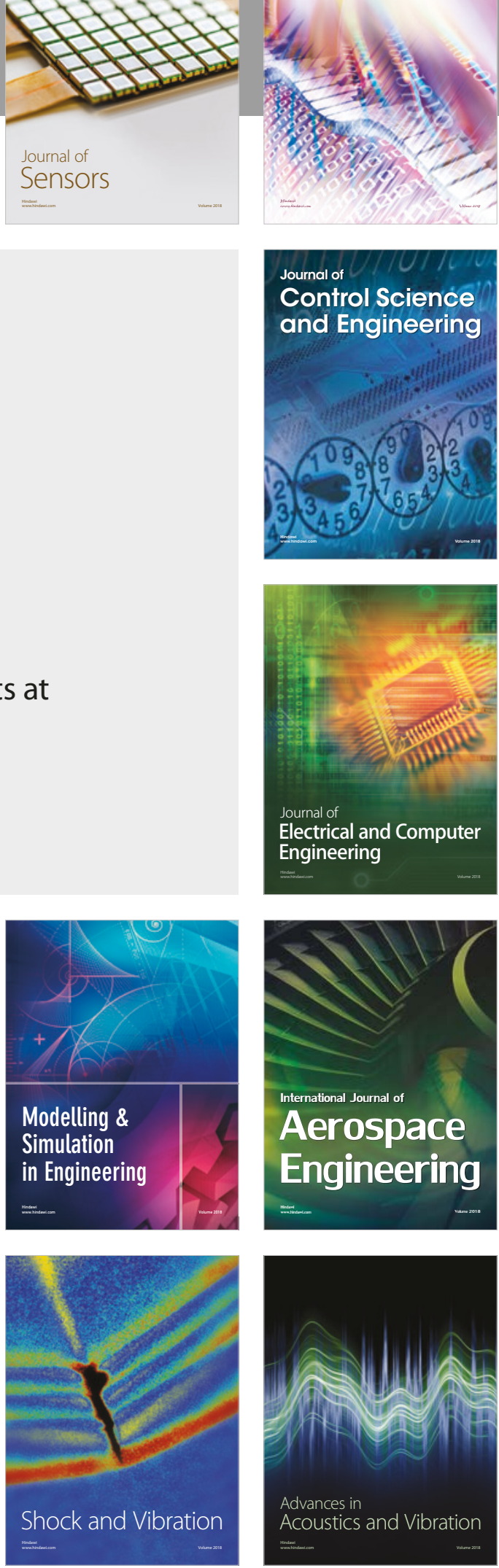